\title{
A genomic and transcriptomic approach for a differential diagnosis between primary and secondary ovarian carcinomas in patients with a previous history of breast cancer
}

\author{
Jean-Philippe Meyniel*+1, Paul H Cottu ${ }^{\dagger 1,2}$, Charles Decraene 2,3,4, Marc-Henri Stern³,5, Jérôme Couturier6,

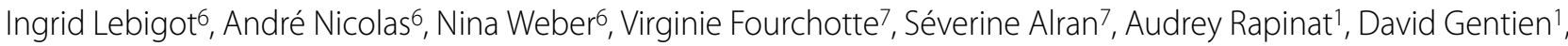 \\ Sergio Roman-Roman'1, Laurent Mignot ${ }^{2}$ and Xavier Sastre-Garau ${ }^{6}$
}

\begin{abstract}
Background: The distinction between primary and secondary ovarian tumors may be challenging for pathologists. The purpose of the present work was to develop genomic and transcriptomic tools to further refine the pathological diagnosis of ovarian tumors after a previous history of breast cancer.

Methods: Sixteen paired breast-ovary tumors from patients with a former diagnosis of breast cancer were collected. The genomic profiles of paired tumors were analyzed using the Affymetrix GeneChip Mapping $50 \mathrm{~K}$ Xba Array or Genome-Wide Human SNP Array 6.0 (for one pair), and the data were normalized with ITALICS (ITerative and Alternative normaLIzation and Copy number calling for affymetrix Snp arrays) algorithm or Partek Genomic Suite, respectively. The transcriptome of paired samples was analyzed using Affymetrix GeneChip Human Genome U133 Plus 2.0 Arrays, and the data were normalized with gc-Robust Multi-array Average (gcRMA) algorithm. A hierarchical clustering of these samples was performed, combined with a dataset of well-identified primary and secondary ovarian tumors.

Results: In 12 of the 16 paired tumors analyzed, the comparison of genomic profiles confirmed the pathological diagnosis of primary ovarian tumor $(n=5)$ or metastasis of breast cancer $(n=7)$. Among four cases with uncertain pathological diagnosis, genomic profiles were clearly distinct between the ovarian and breast tumors in two pairs, thus indicating primary ovarian carcinomas, and showed common patterns in the two others, indicating metastases from breast cancer. In all pairs, the result of the transcriptomic analysis was concordant with that of the genomic analysis.

Conclusions: In patients with ovarian carcinoma and a previous history of breast cancer, SNP array analysis can be used to distinguish primary and secondary ovarian tumors. Transcriptomic analysis may be used when primary breast tissue specimen is not available.
\end{abstract}

\section{Background}

Malignant ovarian tumors comprise a wide and heterogeneous collection of primary and secondary tumors. In patients who had previously developed a breast cancer, the differential diagnosis between primary ovarian carcinoma and metastases from breast cancer may be some-

\footnotetext{
* Correspondence: jean-philippe.meyniel@curie.fr

1 Institut Curie, Department of Translational Research, 26 rue d'Ulm, 75248

Paris, Cedex 05, France

+ Contributed equally

Full list of author information is available at the end of the article
}

times challenging, while it is mandatory to ensure the optimal care for patients. Indeed, metastatic spread and biological pattern differ between metastatic breast cancer and primary ovarian carcinoma. Metastatic breast cancer assessment requires whole body CT-scan, bone scan, and CA 15-3 measurement. Primary ovarian carcinoma assessment requires extensive intra-abdominal exploration and CA-125 measurement. Moreover, the prognosis of metastatic breast cancer and primary ovarian carcinoma widely differs, with a median progression-free survival ranging from 20 to 40 months, and from 9 to 30 
months, respectively $[1,2]$. Most importantly, therapeutic options are very different. Usual therapies for advanced breast cancer may combine or alternate hormonotherapy, chemotherapy regimens, and targeted therapies according to the tumor profile. Conversely, medical therapeutic options for ovarian cancer are scarce, based on paclitaxelcarboplatin combination. Other drugs have been shown to provide minor benefits to the patients, and targeted therapies are only entering early clinical trials.

Surgery is required to provide a thorough exploration of the abdominal cavity, to remove malignant ovarian lesions, to obtain a diagnosis, which is crucial for prognosis, and to plan adequate treatment [3]. In surgical series, ovarian metastases from other primary cancers represent $5 \%$ to $20 \%$ of all ovarian cancers [3-5]. Metastatic lesions to the ovaries are more commonly seen from primary colon cancer, appendiceal, and breast carcinomas. However, there are few clinical or pathological features that make possible to arrive at a differential diagnosis between primary and secondary tumors [6,7].

BRCA1 and BRCA2 mutation carriers have an increased risk of primary breast and ovarian tumors [8], whereas patients with an infiltrating lobular carcinoma (ILC) of the breast are more likely to develop secondary ovarian metastases $[9,10]$. To date, pathological examination remains the cornerstone of the differential diagnosis between primary ovarian tumor and ovarian metastases. In case of metastatic lesions, the involvement of ovaries is more often bilateral, and associated with ascites [3-5]. In those tumors, the pathological feature is more often a stromal rather than a serous invasion, suggesting a metastatic diffusion through blood and lymphatic vessels [5]. In that case, the differential diagnosis is of paramount importance. Several approaches have been developed to discriminate between primary and secondary ovarian cancers. Among them, immunohistochemistry (IHC) has evaluated diagnostic markers, presumably able to support the diagnosis, such as PAX8, a transcription factor for organogenesis of Müllerian system, or Wilms tumor suppressor gene (WT1) whose expression is regulated by PAX8 $[11,12]$. However, only limited series, without validation data, have been reported so far. We speculate that genomic analysis may represent an alternative approach, which could also add some robustness to PAX8 IHC data. To our knowledge, only one study has evaluated high throughput genomic analyses to refine the diagnosis of ovarian lesions following an adenocarcinoma of the endometrium [13]. In a series of nine patients, using $19 \mathrm{k}$ comparative genomic hybridization $(\mathrm{CGH})$ arrays, the authors established that, in three cases out of nine for which the pathological diagnosis was equivocal, the genomic analyses were able to provide a clear diagnosis. In this series, no transcriptomic data were reported.
In the present work, we proposed to develop genomic and transcriptomic approaches to discriminate between primary and secondary ovarian tumors after breast cancer when the pathological diagnosis was equivocal. For this purpose, we characterized genomic and transcriptomic profiles in paired breast and ovarian tumors diagnosed in a same patient, and compared genomic data with clinical and pathological characteristics.

\section{Methods}

\section{Clinical cases and data}

Sixteen patients who had developed an infiltrating breast carcinoma and a subsequent ovarian tumor, and for whom frozen tissues were available for both tumors were included in the study. Breast tumors were classified according to the American Joint Committee on Cancer (AJCC)/Union Internationale Contre le Cancer (UICC) staging system and usual pathological parameters [14]. Ovarian tumors were classified according to the Fédération Internationale de Gynécologie Obstétrique (FIGO) staging system.

Information about surgical and systemic treatments, and the occurrence of other metastatic sites were collected. The clinical data were reviewed by two medical oncologists (PHC, LM). Pathological data were blindly reviewed by two pathologists (NW, XSG), and, in case of discrepancy, a consensus was achieved. To ensure an independent data extraction, all procedures were conducted separately by reviewers, and samples were analyzed without knowledge of their supposed status (i.e. primary tumor, or metastasis).

This study was approved by the Institutional Review Board and Ethics committee. Patients were informed that their biological samples could be used for research purposes and that they had the right to refuse if they so wished.

\section{Immunohistochemical analysis}

Immunohistochemical analysis was performed on formalin-fixed paraffin-embedded tissue sections (depth: 3 $\mu \mathrm{m})$. The samples were deparaffinized and pretreated in EDTA buffer at $\mathrm{pH} 9\left(40\right.$ minutes at $\left.97^{\circ} \mathrm{C}\right)$, and then hydrated in PBS solution for 5 minutes. Then, the rabbit polyclonal anti-PAX8 antibody (Protein Tech Group Inc., Chicago, IL, USA) was applied (dilution: 1/200), and samples were incubated overnight at $4^{\circ} \mathrm{C}$. The endogenous peroxidase activity was blocked with hydrogen peroxide. A second antibody directed against the primary antiPAX8 antibody and coupled with a peroxidase polymer Envision+ (Dako, Trappes, France) was applied for 30 minutes. Then, the peroxidase was revealed during a 10minute incubation with a di-aminobenzidine solution (DAB Dako K3468). Finally, samples were counterstained 
with haematoxylin ( 2 minutes), and mounted with permanent media.

\section{DNA and RNA extraction and preparation for microarray experiment}

Tumor DNA and RNA were provided by the Biological Resource Center of the Institut Curie. Prior to DNA and RNA isolation, a tissue section of tumor fragments was performed and stained with hematoxylin and eosin to evaluate tumor cellularity. All analyzed tumors had more than $50 \%$ of tumoral cells on the tissue section. The DNA was extracted from frozen tumor samples using a standard phenol/chloroform procedure. The total RNA was isolated using TRIzol reagent (Invitrogen, Cergy-Pontoise, France) in accordance with the manufacturers' instructions. The concentration of RNA was measured by absorbance at $260 \mathrm{~nm}$. The quality of each RNA sample was determined with Agilent 2100 bioanalyzer. RNAs were processed on chips only if the following criteria were fulfilled: RIN (a measure of RNA quality) $\geq 7.6$, $(28 \mathrm{~S} / 18 \mathrm{~S}) \geq 1.8,(260 \mathrm{~nm} / 230 \mathrm{~nm}) \geq 1.8$, and $(260 \mathrm{~nm} / 280$ $\mathrm{nm}) \geq 1.8$. Targets were prepared according to Affymetrix (Affymetrix Inc., Santa Clara, CA, USA) One Cycle Synthesis protocol, starting from $2 \mu \mathrm{g}$ of total RNA. Targets were hybridized to GeneChip Human Genome U133 plus 2.0 Arrays if yield and size of targets were reached. Twenty micrograms of complementary RNA, with a specific size distribution were used to hybridize GeneChip Human Genome U133 plus 2.0 Array.

Regarding DNA, the quality was assessed on agarose gel, if a smear was observed instead of a band, the sample was discarded. A 250-ng genomic DNA was used to generate targets according to the GeneChip Mapping $50 \mathrm{~K}$ Xba protocol or Genome-Wide Human SNP Array 6.0 protocol. Targets were prepared if $45 \mu \mathrm{g}$ of amplified DNA were available and if their size was between 250 and $2,000 \mathrm{bp}$, and hybridized according to manufacturer's recommendations.

\section{0 k SNP Array and SNP6.0 Array data analysis}

Intensity signals data from Genechip Human Mapping $50 \mathrm{~K}$ Xba Array or Genome-Wide Human SNP Array 6.0 were normalized and analyzed using ITALICS (ITerative and Alternative normaLIzation and Copy number calling for affymetrix Single nucleotide polymorphism [SNP] arrays) algorithm [15] or Partek Genomic Suite (Partek Inc., St Louis, MO, USA), respectively. The detection and determination of genomics events (gains, losses, amplifications and breakpoints) was performed using GLAD (Gain and Loss Analysis of DNA) software [16] for GeneChip Human Mapping $50 \mathrm{~K}$ Xba Array, and Genomic Segmentation algorithm of Partek Genomic Suite for Genome-Wide Human SNP Array 6.0. Single nucleotide polymorphisms with smoothing value lower and greater than $2 \pm 0.28$ were considered as loss and gain, respectively. The profiles were visualized with the VAMP software [17] or Partek Genomic Suite.

\section{Gene expression data analysis}

A series of 89 ovarian primary tumors and 36 ovarian metastases from breast cancer with a clear pathological diagnosis was used to establish a reference hierarchical tree. All these samples were provided by the Resource Biological Center of the Institut Curie and the chips were processed and hybridized in our laboratory (Department of Translational research). The dataset is publicly available on GEO http://www.ncbi.nlm.nih.gov/geo/ under accession number GSE20565. RNAs were prepared according to the manufacturer's instructions, and were hybridized onto Affymetrix GeneChip Human Genome U133 plus 2.0 Arrays. Transcriptomic data were normalized with gc-Robust Multi-array Average (gcRMA) algorithm [18], using Partek Genomic Suite (Partek Inc., St Louis, MO, USA). Unsupervised hierarchical clustering of tumor samples was done using Partek Genomic Suite software with standard Pearson's correlation as similarity measure, and Ward's method as linkage criteria. The IQR (a measure of the dispersion of each probe set intensity value across all samples) was set in order to have 2000 probe sets. First, the clustering was performed on this set of reference samples (89 primary tumors and 36 ovarian metastases), then the 16 ovarian samples with ambiguous diagnosis were introduced in the dataset and the clustering was performed.

\section{Clustering}

Validation of the reference hierarchical tree was performed using $\mathrm{R}$ environment and the clusterStab package [19]. This package assessed the number of reliable clusters and the stability of the hierarchical clustering with a re-sampling approach whereby randomly selected subsets of samples (70\% each round) are repeatedly clustered. The extent of similarity between the resulting clusters was examined and measured by the Jaccard coefficient ranging from zero (no similarity) to one (identical clustering). We used this strategy for a number of clusters ranging from 2 to 8 , and we compared the results of Jaccard distribution. Enrichment of values equal or close to one indicated adequate choices of metric, agglomeration method and number of clusters. The algorithm was run with the commonly used metrics (Euclidean and Pearson correlation), and the commonly used agglomeration methods (average and Ward's method). The script of the function was adapted in order to use the Pearson correlation coefficient as metric (not implemented in the Bioconductor package). We used the Hmisc package from Bioconductor to calculate this correlation between samples. 
The use of clusterStab package showed that the best reliability of the number of clusters was detected when using Pearson correlation coefficient as metric, Ward's method as agglomeration method, and $\mathrm{k}=2$ clusters (Figure 1).

\section{Results}

\section{Patient and tumor characteristics}

The main characteristics of the sixteen patients and their tumors are summarized in Table 1 . The median age at diagnosis of breast cancer was 48 years (range, 32 to 69 years), and at diagnosis of ovarian tumor was 54 years (range, 38 to 71 years). The median time interval between breast cancer and ovarian tumor diagnosis was 73 months (range, 0 to 150 months). One patient had an ipsilateral breast relapse contemporaneous to the ovarian tumor (case \#2). All patients had a breast surgery plus axillary dissection. Only two patients did not receive any systemic therapy. Other patients received a systemic treatment consisting of adjuvant chemotherapy, tamoxifen alone or chemoendocrine therapy according to institutional guidelines. All patients were irradiated according to our institutional guidelines. At a median follow-up of 39 months after the diagnosis of ovarian tumor (range, 1 to 87 months), five patients were still alive, among whom four had no evidence of disease. The median overall survival from breast cancer diagnosis was 78 months, and 29 months from ovarian tumor diagnosis.
The pathological features of breast and ovarian tumors are presented in Table 2. For breast tumors, the expression of HER2 was assessed (data not shown) as negative in five patients, and was not available for the eleven remaining patients. HER2 is a marker of aggressiveness, the overexpression of HER2 gene increases the cellular growth and metastatic potentialities. For cases number 6 , 11, 15 and 16, the clinical and pathological analyses were not able to draw a definite conclusion regarding the ovarian tumor diagnosis (Table 3) due to the unspecific clinical presentation (i.e.: ovarian carcinoma) or pathological presentation (poorly differentiated carcinoma which could be compatible with either primary or secondary ovarian tumor). Noteworthy, three of these four patients developed previously a non-lobular breast carcinoma (infiltrating ductal carcinoma in two, undifferentiated in one). No anti-PAX8 staining was observed in primary breast carcinoma (data not shown). For the sixteen ovarian tumors, PAX8 was deemed positive in six cases, and negative in ten cases. Based on clinical and pathological characteristics, two patients were treated as metastatic breast cancer, and two as primary ovarian cancer.

\section{SNP array analysis}

In four cases out of sixteen ovarian tumors, the pathological diagnosis was uncertain (Table 3). The comparison of mammary and ovarian genomic profiles was first established in the twelve available pairs with a clear diagnosis.

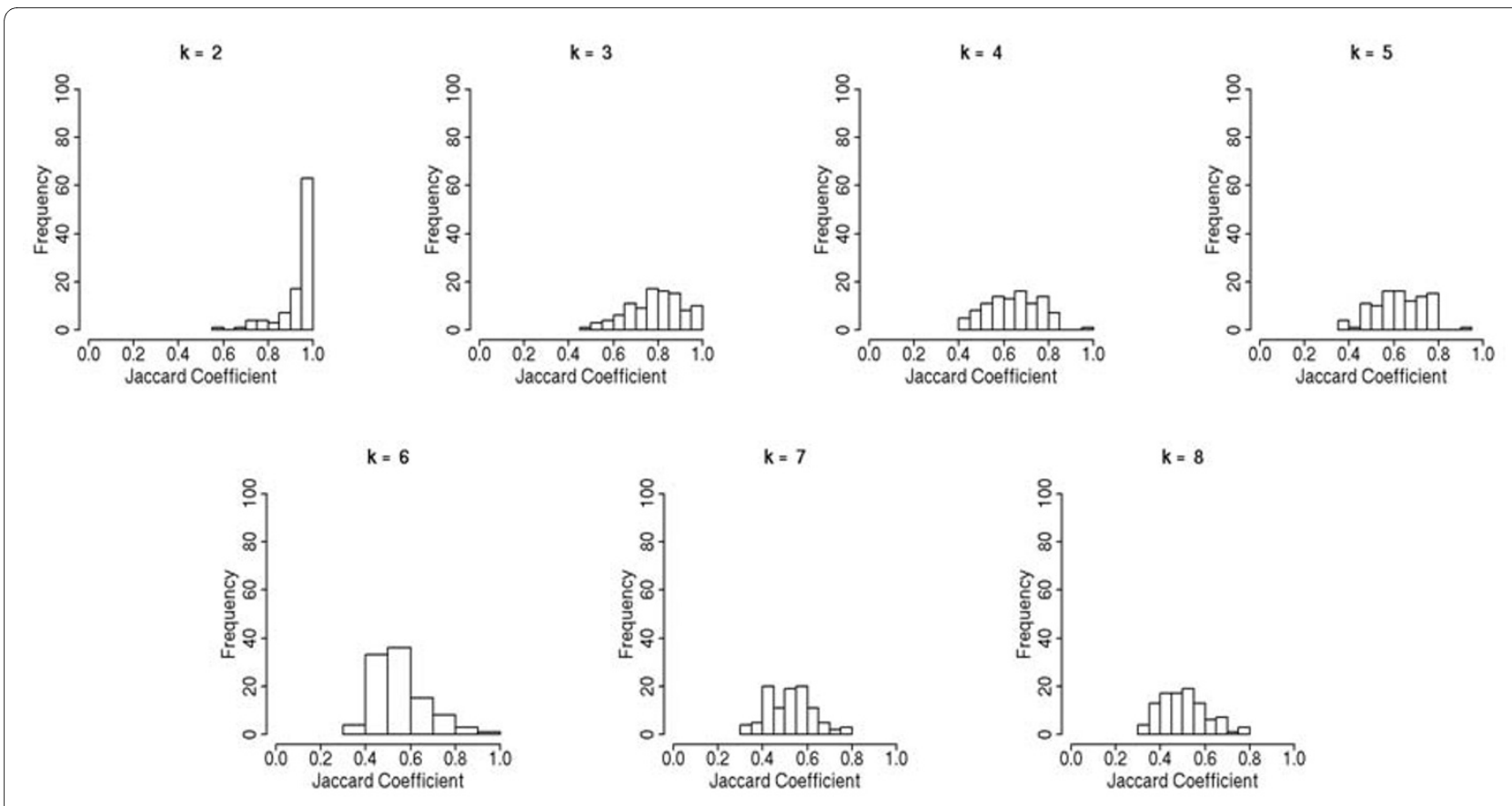

Figure 1 Distributions of Jaccard coefficients, for a number of clusters ranging from $k=2$ to $k=8$, derived from 100 independent random samplings of tumors. Distributions were presented only if Pearson correlation coefficient and Ward's method were used. The proportion of samples used for re-sampling was 0.7 . For $\mathrm{k}=2$ clusters, the largest proportion of values near one indicated that tumors set up two stable clusters. 
Table 1: Demographics, treatment, characteristics of breast and ovarian tumors, and disease outcome of the sixteen patients

\begin{tabular}{|c|c|c|c|c|c|c|c|c|c|c|}
\hline \multirow[b]{2}{*}{ Case \# } & \multirow[b]{2}{*}{$\begin{array}{l}\text { BRCA } \\
\text { status }\end{array}$} & \multicolumn{4}{|c|}{ Breast tumor } & \multicolumn{4}{|c|}{ Ovarian tumor } & \multirow[b]{2}{*}{ Outcome } \\
\hline & & $\begin{array}{l}\text { Age at } \\
\text { diagnosis }\end{array}$ & Stage & $\begin{array}{c}\text { Local } \\
\text { treatment }\end{array}$ & $\begin{array}{l}\text { Systemic } \\
\text { treatment }\end{array}$ & $\begin{array}{l}\text { Age at } \\
\text { diagnosis }\end{array}$ & $\begin{array}{c}\text { Time interval } \\
\text { from BC (months) }\end{array}$ & $\begin{array}{l}\text { FIGO } \\
\text { stage }\end{array}$ & Metastatic site & \\
\hline 1 & ND & 45 & III & M & CT-T & 48 & 34 & IV & Liver & DOD \\
\hline 2 & ND & 48 & III & M & CT & 48 & $73^{*}$ & IV & Liver & DOD \\
\hline 3 & ND & 45 & III & $\mathrm{BCS}$ & CT-T & 57 & 150 & IV & Bone, liver & DOD \\
\hline 4 & ND & 59 & 1 & $\mathrm{BCS}$ & No & 70 & 130 & IV & $\begin{array}{l}\text { Peritoneal } \\
\text { carcinomatosis, } \\
\text { axillary LN }\end{array}$ & DOD \\
\hline 5 & ND & 57 & III & M & $\mathrm{T}$ & 58 & 20 & Illc & NA & DOD \\
\hline 6 & ND & 49 & III & M & CT-T & 52 & 33 & Illle & NA & DOD \\
\hline 7 & BRCA2 m & 66 & 1 & $\mathrm{BCS}$ & CT & 67 & 19 & Ic & NA & NED \\
\hline 8 & $\begin{array}{l}\text { BRCA1 wt, } \\
\text { BRCA2 uk }\end{array}$ & 46 & III & $\mathrm{BCS}$ & CT-T & 46 & 0 & Ila & NA & DOD \\
\hline 9 & ND & 34 & III & $M$ & CT-T & 45 & 133 & IV & $\begin{array}{c}\text { Peritoneal } \\
\text { carcinomatosis, } \\
\text { supraclavicular } \\
\text { LN, brain }\end{array}$ & SD \\
\hline 10 & ND & 47 & III & M & $\mathrm{T}$ & 54 & 83 & IIIC & NA & NED \\
\hline 11 & BRCA1 m & 69 & III & M & CT & 71 & 24 & Ila & NA & DOD \\
\hline 12 & $\begin{array}{l}\text { Familial } \\
\text { history a }\end{array}$ & 47 & III & M & CT-T & 54 & 79 & IIIC & NA & DOD \\
\hline 13 & ND & 50 & 1 & BCS & No & 57 & 87 & IIC & NA & NED \\
\hline 14 & ND & 57 & II & $\mathrm{BCS}$ & $\mathrm{T}$ & 64 & 82 & IIC & NA & NED \\
\hline 15 & $\begin{array}{l}\text { BRCA1 wt, } \\
\text { BRCA2 wt }\end{array}$ & 32 & III & $M$ & $\mathrm{CT}$ & 38 & 74 & IV & Pleura & DOD \\
\hline 16 & ND & 48 & $\|$ & $\mathrm{BCS}$ & CT & 49 & 18 & IIIC & NA & DOD \\
\hline
\end{tabular}

In the subset of seven metastatic samples, one array was not usable for analysis (signal intensity too noisy on the chip). Among the six remaining samples, similar genetic events were found in mammary and ovarian tumors. The alterations found in breast samples were observed in ovarian samples, whereas some additional alterations were observed in the ovarian samples as visually depicted in Figure 2. Moreover, the breakpoints detected in the mammary tumor were also found at the same position in the ovarian sample. Additional breakpoints were also found in this subset of ovarian tumors. These observations led to the conclusions that these six ovarian tumors were metastases of the primary breast cancer.
In the other subset of five samples considered as primary ovarian carcinoma, no overlap of genomic alterations was found between mammary and ovarian samples (Figure 3). As well, no common position for breakpoints was observed, thus confirming the primary status of the ovarian tumors (Table 3 ).

Then, molecular diagnosis was performed on the four ambiguous tumors. Two of them were classified as metastases from breast cancer as similar genetic events were observed in the primary tumor and its ovarian counterpart (see Additional Files 1 and 2). The two remaining tumors were classified as primary tumors as their genetic profiles were strikingly different from those of the original breast tumors (see Table 3 and Additional Files 3 and $4)$. 
Table 2: Pathological features of breast and ovarian tumors

\begin{tabular}{|c|c|c|c|c|c|c|c|c|}
\hline \multirow[b]{2}{*}{ Case \# } & \multicolumn{2}{|c|}{ Histology } & \multicolumn{2}{|c|}{ Grade } & \multicolumn{2}{|c|}{ ER } & \multicolumn{2}{|c|}{ PgR } \\
\hline & Breast & Ovary & Breast & Ovary & Breast & Ovary & Breast & Ovary \\
\hline 1 & ILC & Lobular carcinoma & I & ND & + & + & + & ND \\
\hline 2 & IDC, then ILC* & Lobular carcinoma & III, then II & ND & + & ND & + & ND \\
\hline 3 & ILC & Lobular carcinoma & 1 & ND & + & + & + & ND \\
\hline 4 & ILC & Lobular carcinoma & ND & ND & + & + & + & - \\
\hline 5 & ILC & Lobular carcinoma & III & ND & + & ND & + & ND \\
\hline 6 & ILC & Lobular carcinoma & $\|$ & ND & + & ND & - & ND \\
\hline 7 & IDC & Serous papillary & III & III & - & ND & - & ND \\
\hline 8 & IDC & Serous papillary & III & ॥ & + & - & + & + \\
\hline 9 & IDC & Poorly differentiated ADK & III & ND & + & ND & + & ND \\
\hline 10 & IDC and ILC (mixed) & Serous papillary & III & III & + & - & + & - \\
\hline 11 & IDC & Poorly differentiated ADK & III & ॥ & - & + & - & + \\
\hline 12 & ILC & Lobular carcinoma & III & ND & + & + & + & ND \\
\hline 13 & IDC & Serous papillary & II & III & + & ND & + & ND \\
\hline 14 & IDC & Clear cell & $\|$ & $\|$ & + & ND & - & ND \\
\hline 15 & IDC & Poorly differentiated ADK & ॥ & ND & + & ND & + & ND \\
\hline 16 & Undifferentiated & Serous papillary & III & III & - & + & - & + \\
\hline
\end{tabular}

\section{Transcriptomic analysis}

Fifteen out of sixteen gene expression profiles of ovarian tumors were obtained. These tumors were included in a set of samples including well-identified primary ovarian tumors $(n=89)$, and ovarian metastases $(n=36)$. All the samples were processed, normalized, and clusterized as described above. Therefore, a hierarchical tree with two main branches was obtained: the first branch gathered all metastatic samples, and the second branch contained all primary tumors but one (Figure 4). The reference hierarchical tree obtained with the 125 reference tumors was not modified when the fifteen studied tumors were added. Two main branches were repeatedly generated with the raising of the tree from 125 to 140 tumors. The fifteen ovarian tumors of the core subset all segregated accordingly to the pathological diagnosis and their genomic profiles (Figure 4, Table 3 ). The tumor \#1, with no SNP array data, clearly segregated with metastatic tumors.

\section{Discussion}

So far, no definitive molecular profiling of ovarian cancer has been published. Molecular characterization is only based on grade, histo-pathological features and some key genes alterations. As well, no high-throughput molecular data on ovarian metastasis have been reported. This study is the first to use a comprehensive genomic analysis to discriminate primary ovarian carcinomas and metastatic ovarian lesions from breast cancer. A similar approach was evaluated to distinguish primary from secondary ovarian tumors after adenocarcinoma of the endometrium [13]. Using a 19 K CGH array, four cases out of nine remained undetermined. In our data set, the pathological status of ovarian lesions was well established in twelve cases. The blind test of these pairs for SNP and transcriptomic analyses was in agreement with the pathological analysis (Table 3). In the four lesions with an ambiguous pathological diagnosis, SNP and transcriptomic analyses permitted to clearly discriminate primary tumor and metastatic disease. This is also the first time that both transcriptomic and genomic analyses were performed, demonstrating a complete overlap of results with these two kinds of analyses.

The purpose of the present work was not to provide a genomic signature, but to give a diagnosis allowing a treatment decision. Conversely to the study of ovarian tumors after adenocarcinoma of the endometrium using low-density $19 \mathrm{k}$ BAC arrays [13], we used high-density $50 \mathrm{~K}$ SNP arrays, showing major chromosomal alterations in all tumors. This method allowed interpretation and 
Table 3: Comparison between pathological analyses, $\mathrm{CGH}$, transcriptomic profiles, and immunohistochemistry profiles

\begin{tabular}{lllll}
\hline Case \# & Pathological analysis & Genomic analysis & Transcriptomic analysis & PAX8 IHC \\
\hline 1 & Metastasis & ND & Metastasis & Negative \\
2 & Metastasis & Metastasis & Metastasis & Negative \\
3 & Metastasis & Metastasis & Metastasis & Negative \\
4 & Metastasis & Metastasis & Metastasis & Negative \\
5 & Metastasis & Metastasis & Metastasis & Negative \\
6 & Plausible metastasis & Metastasis & Metastasis & Pegative \\
7 & Primary tumor & Primary tumor & Primary tumor & Positive \\
8 & Primary tumor & Primary tumor & Primary tumor & Negative \\
9 & Metastasis & Metastasis & Metastasis & Positive \\
10 & Primary tumor & Primary tumor & Primary tumor & Positive \\
11 & Plausible primary tumor & Primary tumor & Primary tumor & Negative \\
12 & Metastasis & Metastasis & Metastasis & Positive \\
13 & Primary tumor & Primary tumor & Primary tumor & Negative \\
14 & Primary tumor & Primary tumor & ND & Negative \\
15 & Plausible metastasis & Metastasis & Metastasis & Positive \\
16 & Plausible primary tumor & Primary tumor & Primary tumor & \\
\hline
\end{tabular}

PAX8: Paired Box 8 gene; $N D$ = not determined; $I H C=$ immunohistochemistry

comparison of the genomic profiles within the fifteen available pairs of breast and ovarian tumors. When most of genomic alterations found in primary breast tumors were included in the ovarian counterpart, a genomic diagnosis of metastases was proposed and then confirmed by the comparison with the histopathological diagnosis. A previous experience of $\mathrm{CGH}$ in metastatic breast cancer has been reported, comparing primary tumor with lymph node metastases, distant metastases or

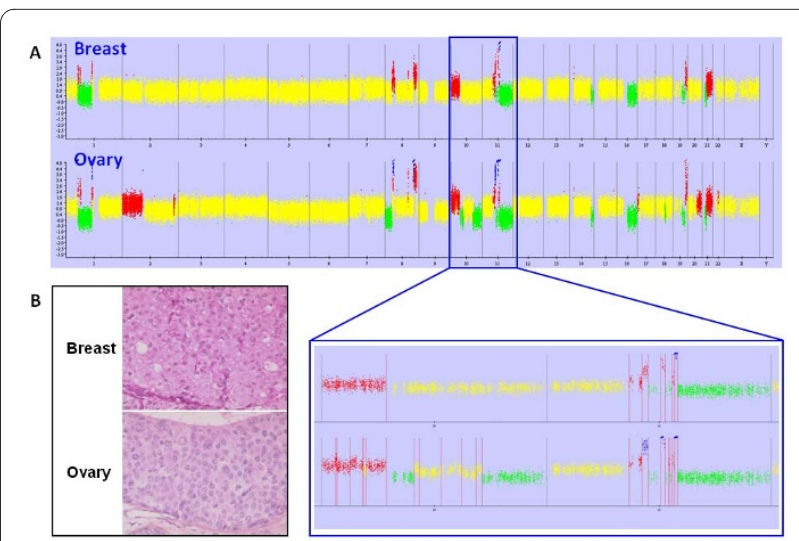

Figure 2 (A) Genomic profile of the breast/ovary sample pair \#9. In the zoomed area, similar altered areas and breakpoint positions (red vertical lines) between mammary and ovarian tumors were detected. Yellow $=$ normal; red $=$ gain; green $=$ loss; blue $=$ amplification. $X$ axis: all chromosomes, $Y$ axis: SNP copy number. (B) Histological sections of breast (upper) and ovarian (lower) tumors local recurrences in sixteen patients [20]. Genomic profiles comparison showed that additional aberrations were detected in the lymph nodes or distant metastases when compared to the primary tumors. These findings suggest that progression from primary breast cancer to metastasis may be associated with the acquisition of further genetic changes. Overall, in the present series, we confirmed these findings and proposed the use of SNP array

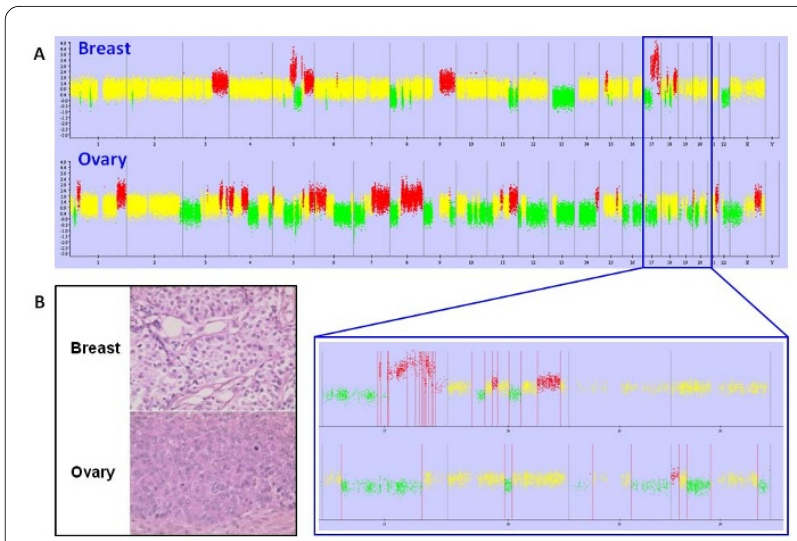

Figure 3 (A) Genomic profile of the breast/ovary sample pair \#8 In the zoomed area, no similarities for altered areas and breakpoint positions (red vertical lines) between mammary and ovarian tumors were detected. Yellow = normal; red = gain; green = loss; blue = amplification. $X$ axis: all chromosomes, Y axis: SNP copy number. (B) Histological sections of breast (upper) and ovarian (lower) tumors 


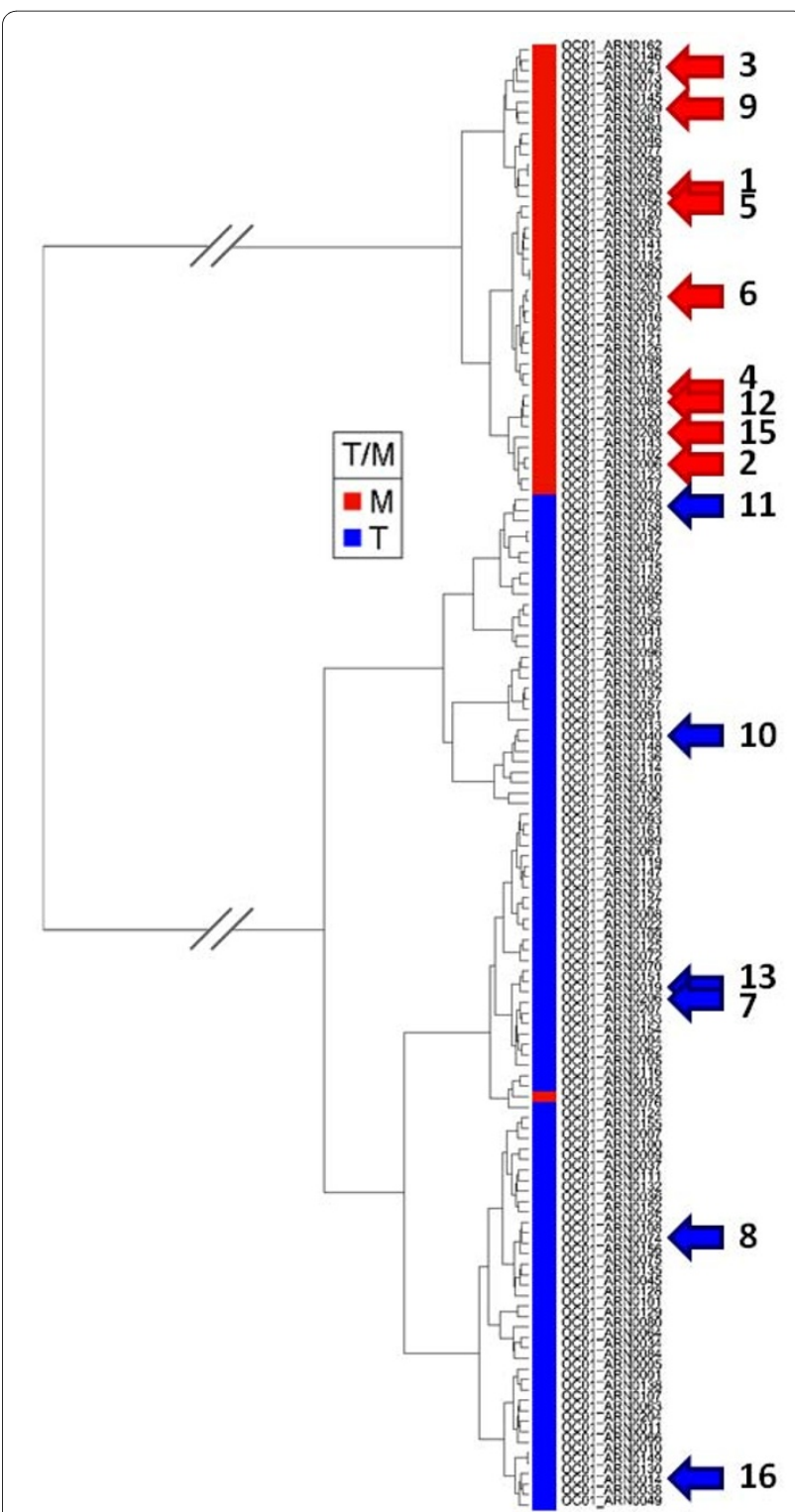

Figure 4 Hierarchical clustering. Pearson's correlation coefficient and Ward's method were used. In red are the ovarian metastases samples, and in blue the ovarian primary tumors. Red and blue arrows localized the ovarian metastases and primary tumor samples, respectively, among the 16 cases included in this study

technology to provide a definite diagnosis in case of equivocal ovarian lesions.

Furthermore, the present study highlighted that the transcriptomic analysis was always in concordance with the pathological diagnosis in the reference set of wellidentified 125 tumors, excepted for one case considered as a metastasis, but clustering with primary tumors (Figure 4). Both analyses (genomic and transcriptomic) were possible in fourteen pairs of tumors, and results were always identical (Table 3). Given the robustness of our reference tree, we have therefore established a dataset of reference that is now available for future research and diagnostic purposes. The main interest of this method is to have at one's disposal a test that does not require the primary tumor to give a diagnosis. Indeed, other authors have reported a similar approach in particular with Carcinomas of Unknown Primary (CUP) [21], suggesting that expression data may help in elucidating the histogenetic origin of metastatic tissue. The future directions might be to enrich our dataset of tumors likely to give ovarian metastases, such as gastric or colorectal carcinomas.

The use of WT1 in IHC analysis seems not to be appropriate to discriminate between primary and secondary tumors. A recent study [22] showed that WT1 is rather dedicated to distinguish between serous and endometrioids ovarian carcinomas. So, in our study, we preferred to use PAX8 staining instead of WT1 staining for the distinction of primary and secondary lesions.

Interestingly, PAX8 IHC status was in concordance with genomic results in the present model of primary ovarian tumors compared to ovarian metastases from breast cancer, except for one patient who had a clear-cell carcinoma. PAX8 is usually highly, but not always, expressed in serous-papillary (96,4\%), endometrioid $(88,9 \%)$ or clear-cell carcinoma (100\%) [11]. In the series of these authors, PAX8 staining was positive for all the clear-cell carcinoma (10/10), so they concluded that PAX8 was particularly useful for the diagnosis of this histotype. But the only one sample which is not stained in our collection is a clear-cell ovarian carcinoma. Moreover, no definite nor consensual criteria for PAX8 IHC positivity has been defined so far, yielding some concern about the reproducibility of their technique. To our opinion, PAX8 IHC does not provide a totally robust result concerning the status of ovarian tumors. In the present series, genomic analyses yielded a definite diagnosis in all cases. In another study concerning mouse model of human ovarian endometrioid adenocarcinoma [23], the authors performed IHC and sequence analysis, in parallel, on some genes implicated in the pathology. For CTNNB1 gene, the results were totally concordant between IHC and sequence analyses. But for other genes, like PTEN and TP53, there were some discrepancies. For example, 12 samples were found to be mutated with IHC for PTEN whereas when the authors examined the sequence, mutations were found only in 6 samples. In this case, they detected 6 false positives with IHC analysis. Also, when they analyzed TP53 mutations, they detected 3 false positives and 2 false negatives between IHC and sequence analyses. These results demonstrate that genomic tools are more appropriate to determine definitively the mutational status of genes. 


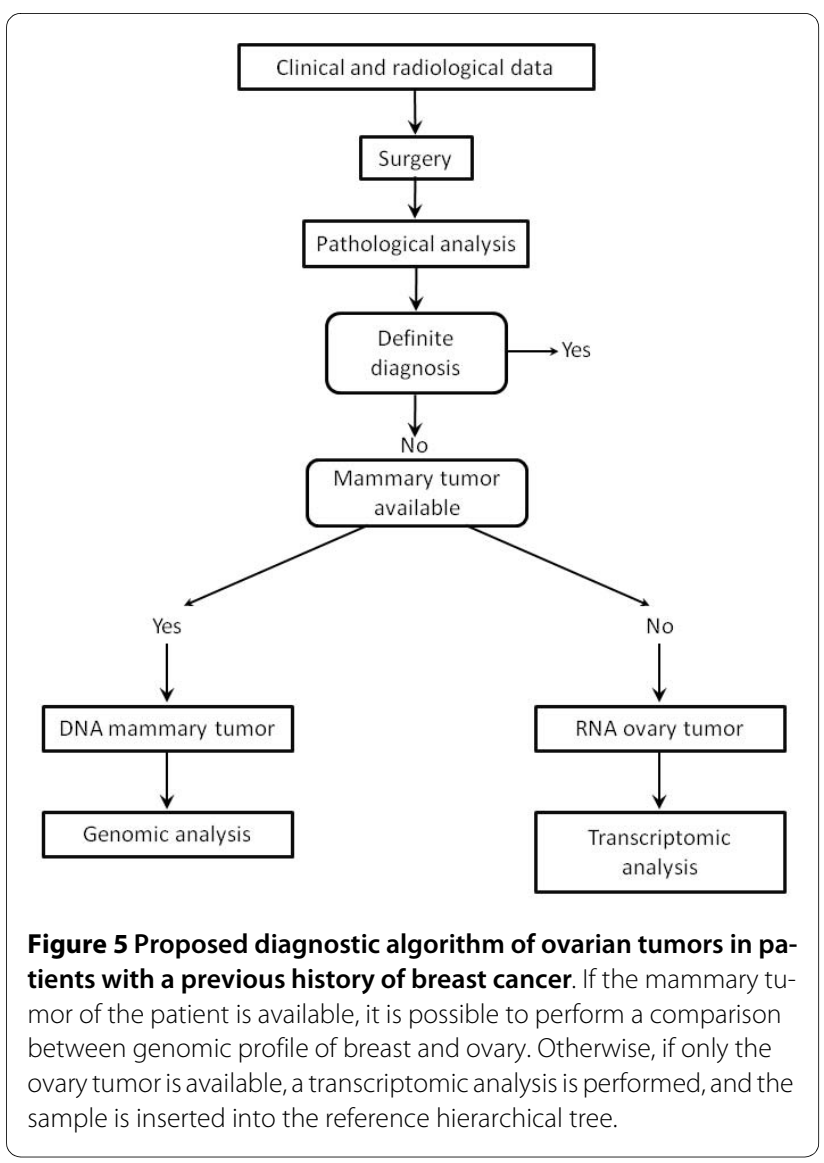

\section{Conclusions}

We established the robustness of SNP and transcriptomic analysis to discriminate primary ovarian tumors and ovarian metastases after primary breast cancer. Noteworthy, all genomic analyses were blindly compared to clinical and pathological characteristics. An algorithm of diagnosis could be considered in the light of these results (Figure 5). The first step remains the pathological analysis. If the primary tumor is not available, which represents the majority of cases, a transcriptomic analysis may be performed. If the primary tumor is available, the SNP array is the preferential test. Of course, these results require further analyses and comparison with other biomarkers.

\section{Additional material}

Additional file 1 Genomic profile of the breast/ovary sample pair \#6 with uncertain diagnosis. The genomic profiles of this pair were performed using Affymetrix GeneChip" Mapping $50 \mathrm{~K}$ Xba Array. The normalization and segmentation methods used for this kind of array were those described in Materials and Methods. Few alterations were detected in the breast and the ovary samples. In the zoomed area, we see that an alteration and breakpoints (red vertical lines) are detected at the same positions in the 2 samples, indicating that the ovary tumor is a metastasis from the breast. Yellow $=$ normal; red $=$ gain; green $=$ loss; blue $=$ amplification. $X$ axis: all chromosomes, $Y$ axis: SNP copy number.
Additional file $\mathbf{2}$ Genomic profile of the breast/ovary sample pair \#15 with uncertain diagnosis. The genomic profiles of this pair were performed using Affymetrix GeneChip "Mapping $50 \mathrm{~K}$ Xba Array. The normalization and segmentation methods used for this kind of array were those described in Materials and Methods. In the zoomed area, we observe that common alterations are found between the breast and the ovary tumors. Moreover, breakpoints (red vertical lines) are detected exactly at the same positions between the 2 samples. So, in that case, the ovary tumor is a metastasis from the breast. Yellow = normal; red = gain; green = loss; blue = amplification. $X$ axis: all chromosomes, $Y$ axis: SNP copy number.

Additional file 3 Genomic profile of the breast/ovary sample pair \#11 with uncertain diagnosis. The genomic profiles of this pair were performed using Affymetrix Genome-Wide Human SNP Array 6.0 Array. The normalization and segmentation methods used for this kind of array were those described in Materials and Methods. Only the first 6 chromosomes are shown but they are representatives of all the alteration profiles observed on the 2 samples. In each chromosome graph, the 2 top profiles represent the chromosomal copy number, the 2 bottom graphs represent the result of genomic segmentation algorithm: red area = gain, blue area = loss. We can observe that no common alterations are detected between the breast and the ovary samples, indicating that the breast and the ovary tumors are both primary tumors.

Additional file 4 Genomic profile of the breast/ovary sample pair \#16 with uncertain diagnosis. The genomic profiles of this pair were performed using Affymetrix GeneChip" Mapping $50 \mathrm{~K}$ Xba Array. The normalization and segmentation methods used for this kind of array were those described in Materials and Methods. Along the chromosomes and in the zoomed area, no common alteration or breakpoint (red vertical lines) position is detected between the breast and the ovary tumors. This result reveals that the ovary tumor is a primary tumor and not a metastasis from the breast. Yellow = normal; red = gain; green = loss; blue = amplification. $X$ axis: all chromosomes, $Y$ axis: SNP copy number.

\section{Abbreviations}

ADK: Adenocarcinoma; AJCC: American Joint Committee on Cancer; BCS: Breast-Conserving Surgery; BC: Breast Cancer; BRCA-1(-2): BReast CAncer-1(-2) gene; CA-125: Carbohydrate Antigen 125; CA 15-3: Carbohydrate Antigen 15-3; CGH: Comparative Genomic Hybridization; CT: ChemoTherapy; CT-scan: Computed Tomography-scan; DNA: DesoxyriboNucleic Acid; DOD: Died Of Disease; EDTA: Ethylene Diamine Tetraacetic; ER: Estrogen Receptor; FIGO: Fédération Internationale de Gynécologie Obstétrique; gcRMA: gc-Robust Multi-array Average; GLAD: Gain and Loss Analysis of DNA; HER2: Human Epidermal Growth Factor Receptor-2; IDC: Infiltrating Ductal Carcinoma; IHC: ImmunoHistoChemistry; ILC: Infiltrating Lobular Carcinoma; IQR: InterQuartile Range; ITALICS: ITerative and Alternative normaLIzation and Copy number calling for affymetrix Snp arrays; LN: Lymph Nodes; m: Mutation; M: Mastectomy; NA: Not Applicable; ND: Not Determined; NED: No Evidence of Disease; PAX8: Paired Box 8 gene; PBS: Phosphate Buffered Saline; PgR: Progesterone Receptor; RIN: RNA Integrity Number; RNA: Ribonucleic Acid; SD: Stable Disease; SNP: Single Nucleotide Polymorphism; T: Tamoxifen; UICC: Union Internationale Contre le Cancer; uk: Unknown; VAMP: Visualization and analysis of Array-CGH, transcriptome and other Molecular Profiles; WT1: Wilms Tumor suppressor gene; WT: Wild Type

\section{Competing interests}

The authors declare that they have no competing interests.

\section{Authors' contributions}

JPM, with the support of CD, AR, DG and SRR, conceived of the study, participated in its design and coordination, carried out the translational and statistical analysis, and helped to draft the manuscript. PHC conceived of the study, participated in its design and coordination and helped to draft the manuscript. PHC and LM carried out the follow-up of patients and reviewed clinical data. JC, IL, AN, NW and XSG carried out the pathologic analysis. VF and SA carried out the surgical procedures. All authors read and approved the final manuscript.

\section{Acknowledgements}

We wish to thank Cécile Reyes and Benoit Albaud, from the Affymetrix Platform of the Institut Curie, for their help in the production and the processing of the 
chips, Blandine Massemin and Michèle Galut for their help in the processing of DNA and RNA samples. Isabelle Chapelle-Marcillac provided editorial assistance in the preparation of the manuscript.

\section{Author Details}

'Institut Curie, Department of Translational Research, 26 rue d'Ulm, 75248 Paris, Cedex 05, France, 2Institut Curie, Department of Medical Oncology, 26 rue d'Ulm, 75248 Paris, Cedex 05, France, 3 Institut Curie, Research Unit, 26 rue d'Ulm, 75248 Paris, Cedex 05, France, ${ }^{4}$ CNRS, UMR144, 26 rue d'Ulm, 75248 Paris, Cedex 05, France, 5INSERM, U830, 26 rue d'UIm, 75248 Paris, Cedex 05, France, ${ }^{6}$ Institut Curie, Department of Pathology, 26 rue d'Ulm, 75248 Paris, Cedex 05, France and 7Institut Curie, Department of Surgery, 26 rue d'UIm, 75248 Paris, Cedex 05, France

\section{Received: 22 October 2009 Accepted: 21 May 2010}

Published: 21 May 2010

\section{References}

1. Singletary $\mathrm{SE}$, Connolly $\mathrm{JL}$ : Breast cancer staging: working with the sixth edition of the AJCC Cancer Staging Manual. CA Cancer J Clin 2006, 56:37-47.

2. Högberg T, Glimelius B, Nygren P: A systematic overview of chemotherapy effects in ovarian cancer. Acta Oncol 2001, 40:340-360.

3. Turan T, Aykan B, Koc S, Boran N, Tulunay G, Karacay O, Erdogan Z, Kose F: Analysis of metastatic ovarian tumors from extragenital primary sites. Tumori 2006, 92:491-495.

4. Mazur MT, Hsueh S, Gersell DJ: Metastases to the female genital tract. Analysis of 325 cases. Cancer 1984, 53:1978-1984.

5. Moore RG, Chung M, Granai CO, Gajewski W, Steinhoff MM: Incidence of metastasis to the ovaries from nongenital tract primary tumors. Gynecol Oncol 2004, 93:87-91.

6. Le Thai N, Lasry S, Brunet M, Harvey P, Rouesse J: Cancer of the ovary after cancer of the breast. 45 cases [Article in French]. J Gynecol Obstet Biol Reprod (Paris) 1991, 20:1043-1048.

7. Young RH: From Krukenberg to today: the ever present problems posed by metastatic tumors in the ovary - Part II. Adv Anat Pathol 2007, 14:149-177.

8. Levy-Lahad E, Friedman E: Cancer risks among BRCA1 and BRCA2 mutation carriers. Br J Cancer 2007, 96:11-15.

9. Harris M, Howell A, Chrissohou M, Swindel RIC, Hudson M, Sellwood RA: A comparison of the metastatic pattern of infiltrating lobular carcinoma and infiltrating duct carcinoma of the breast. Br J Cancer 1984, 50:23-30.

10. Sastre-Garau X, Jouve M, Asselain B, Vincent-Salomon A, Beuzeboc $P$, Dorval T, Durand JC, Fourquet A, Pouillart P: Infiltrating lobular carcinoma of the breast. Clinicopathologic analysis of 975 cases with reference to data on conservative therapy and metastatic patterns. Cancer 1996, 77:113-120.

11. Nonaka D, Chiriboga L, Soslow RA: Expression of pax8 as a useful marker in distinguishing ovarian carcinomas from mammary carcinomas. Am J Surg Pathol 2008, 32:1566-1571.

12. Tornos C, Soslow R, Chen S, Akram M, Hummer AJ, Abu-Rustum N, Norton L, Tan LK: Expression of WT1, CA 125, and GCDFP-15 as useful markers in the differential diagnosis of primary ovarian carcinomas versus metastatic breast cancer to the ovary. Am J Surg Pathol 2005, 29:1482-1489

13. Mhawech-Fauceglia P, Rai H, Nowak N, Cheney RT, Rodabaugh K, Lele S, Odunsi K: The use of array-based comparative genomic hybridization (a-CGH) to distinguish metastatic from primary synchronous carcinomas of the ovary and the uterus. Histopathology 2008 , 53:490-495

14. Goldhirsch A, Wood WC, Gelber RD, Coates AS, Thürlimann B, Senn HJ: Progress and promise: highlights of the international expert consensus on the primary therapy of early breast cancer 2007. Ann Oncol 2007, 18:1133-144

15. Rigaill G, Hupé P, Almeida A, La Rosa P, Meyniel JP, Decraene C, Barillot E: ITALICS: an algorithm for normalization and DNA copy number calling for Affymetrix SNP arrays. Bioinformatics 2008, 24:768-774.

16. Hupé P, Stransky N, Thiery JP, Radvanyi F, Barillot E: Analysis of array CGH data: from signal ratio to gain and loss of DNA regions. Bioinformatics 2004, 20:3413-3422.
17. La Rosa P, Viara E, Hupé P, Pierron G, Liva S, Neuvial P, Brito I, Lair S, Servant N, Robine N, Manié E, Brennetot C, Janoueix-Lerosey I, Raynal V, Gruel N, Rouveirol C, Stransky N, Stern MH, Delattre O, Aurias A, Radvanyi F, Barillot E: VAMP : Visualization and analysis of array- $\mathrm{CGH}$, transcriptome and other molecular profiles. Bioinformatics 2006, 22:2066-2073.

18. Wu Z, Irizarry RA: Preprocessing of oligonucleotide array data. Nat Biotechnol 2004, 22:656-658.

19. MacDonald JW, Ghosh D: COPA--cancer outlier profile analysis. Bioinformatics 2006, 22:2950-2951.

20. Nishizaki T, DeVries S, Chew K, Goodson WH, Ljung BM, Thor A, Waldman FM: Genetic alterations in primary breast cancers and their metastases: direct comparison using modified comparative genomic hybridization. Genes Chromosomes Cancer 1997, 19:267-272.

21. Monzon FA, Lyons-Weiler M, Buturovic L, Rigl T, Henner WD, Sciulli C, Dumur Cl, Medeiros F. Anderson GG: Multicenter validation of a 1,550gene expression profile for identification of tumor tissue of origin. $J$ Clin Oncol 2009, 27:2503-2507.

22. Madore J, Ren F, Filali-Mouhim A, Sanchez L, Köbel M, Tonin PN, Huntsman D, Provencher DM, Mes-Masson AM: Characterisation of the molecular differences between ovarian endometrioid carcinoma and ovarian serous carcinoma. J Pathol 2010, 220:382-400.

23. Wu R, Hendrix-Lucas N, Kuick R, Zhai Y, Schwartz DR, Akyol A, Hanash S, Misek DE, Katabuchi H, Williams BO, Fearon ER, Cho K: Mouse model of human ovarian endometrioid adenocarcinoma based on somatic defects in the Wnt/ $\beta$-catenin and PI3K/Pten signaling pathways. Cancer Cell 2007, 11:321-333.

\section{Pre-publication history}

The pre-publication history for this paper can be accessed here: http://www.biomedcentral.com/1471-2407/10/222/prepub

\section{doi: 10.1186/1471-2407-10-222}

Cite this article as: Meyniel et al., A genomic and transcriptomic approach for a differential diagnosis between primary and secondary ovarian carcinomas in patients with a previous history of breast cancer BMC Cancer 2010, 10:222

\section{Submit your next manuscript to BioMed Central and take full advantage of:}

- Convenient online submission

- Thorough peer review

- No space constraints or color figure charges

- Immediate publication on acceptance

- Inclusion in PubMed, CAS, Scopus and Google Scholar

- Research which is freely available for redistribution
C Biomed Central 